\title{
MAGIC observation and broadband characterization of the remarkably bright flares of 1ES $1959+650$ in 2016
}

\author{
Wrijupan Bhattacharyya, ${ }^{a}$ Mitsunari Takahashi ${ }^{* b}$ and Masaaki Hayashida ${ }^{c}$ on behalf \\ of the MAGIC and Fermi-LAT collaboration \\ ${ }^{a}$ Deutsches Elektronen-Synchrotron (DESY), Germany \\ ${ }^{b}$ Japanese MAGIC Consortium, Japan \\ ${ }^{c}$ Department of Physics, Rikkyo University, Japan \\ E-mail: wrijupan.bhattacharyya@desy.de, takhsmeicrr.u-tokyo.ac.jp, \\ masaaki.hayashida@rikkyo.ac.jp
}

1ES $1959+650$ is a bright TeV high-frequency peaked BL Lac object exhibiting interesting features like broad high energy peak in the spectral energy distribution (SED) and "orphan" TeV flares, that are difficult to interpret using a conventional one-zone synchrotron self-Compton (SSC) scenario. We report the results from MAGIC observations in 2016 along with the multiwavelength data from Fermi-LAT and Swift instruments. An exceptionally high very-high-energy (VHE) flux reaching $\sim 3$ times the Crab Nebula flux was measured by MAGIC on 13th, 14th June and 1st July 2016. It is the highest flux level observed since 2002. During these flares, the second SED peak lies in the VHE domain, and the spectrum extends up to several $\mathrm{TeV}$ energies. The LAT and X-ray spectra are also hard with index $<2$.On 13th June, the source showed fast variabilities in the VHE flux with timescales less than an hour. A simple one-zone SSC model can satisfactorily describe the data during the high VHE states requiring a Doppler factor $\delta \leq 30-60$. Alternatively, the SED can be explained by synchrotron radiation of relativistic protons with a reasonable jet power $L_{\text {jet }} \sim 10^{46} \mathrm{erg} \cdot \mathrm{s}^{-1}$ under the requirement of high magnetic fields $B \sim 100$ $\mathrm{G}$ and maximum proton energy $\sim$ few EeV. The theoretical models imply that detecting neutrino emission from the source during a similar flare with the extreme VHE flaring period of 2016 is difficult.

36th International Cosmic Ray Conference -ICRC2019-

July 24th - August 1st, 2019

Madison, WI, U.S.A.

\footnotetext{
* Speaker.

† https: / /magic.mpp.mpg.de/. For collaboration list see PoS(ICRC2019)1177

${ }^{\ddagger}$ For collaboration list see PoS(ICRC2019)1177
} 


\section{Introduction}

1ES $1959+650$ is a well-known high-frequency peaked BL-Lac (HBL) object located at redshift $z=0.048$ [1]. [2] reported an "orphan flare" on June 4 2002, a bright burst in the very-highenergy (VHE; $E>100 \mathrm{GeV}$ ) $\gamma$-ray band without a simultaneous counterpart at longer wavelengths. In general, flux in the X-ray and $\gamma$-ray are correlated, which is usually explained by standard leptonic emission models. The exception are "orphan flares" (such as the one observed on June 4 2002 [2], bright bursts in the very-high-energy (VHE; E > $100 \mathrm{GeV}$ ) $\gamma$-ray band without a simultaneous counterpart at longer wavelengths. The lack of such a correlation challenges the synchrotron self-Compton (SSC) interpretation of the VHE emission. On the other hand, X-ray flares with low $\gamma$-ray states [3] and simultaneous flaring in the VHE and X-ray [2] are also observed.

The first potential association between a high-energy neutrino event and the flaring blazar TXS $0506+056$ ([4]) has inaugurated a new era in multi-messenger astronomy. 1ES 1959+650 is also an interesting candidate for neutrino point-source search by the IceCube Neutrino Observatory. A hint of high-energy neutrinos spatially and temporally coincident with flares of 1ES 1959+650 was reported with the Antarctic Muon And Neutrino Detector Array although the detections were not statistically significant [5]. A recent IceCube analysis for 8 years of data, is however consistent with the background-only hypothesis. Since 2015 the source became active across several energy bands, remarkably in optical [6], X-rays [7, 8] and also $\gamma$ rays $[9,10,11]$. The lack of correlation between X-ray and VHE $\gamma$ rays during flares as well as the possible detection of neutrino emission challenges the standard SSC model of blazar emission.

In this contribution, we focus on two flaring nights of 1ES 1959+650 observed by multiwavelenghth (MWL) instruments in 2016 when the source was in an active state. The Major Atmospheric Gamma Imaging Cherenkov (MAGIC) telescopes detected two major VHE $\gamma$-ray flares on 13th and 14th June 2016. The flares of 1ES 1950+650 are particularly interesting since the proximity and brightness of the source enable us to perform detailed spectral measurements up to $\mathrm{TeV}$ energies, study the flux variability, test emission models related to the origin of the VHE $\gamma$ rays and inspect their connection to cosmic-ray and neutrino production.

\section{Observation and data analysis}

\subsection{MAGIC Telescopes}

MAGIC is a system of two Imaging Atmospheric Cherenkov Telescopes (IACTs) [12]. The standard trigger energy threshold for low zenith angle observations is $\sim 50 \mathrm{GeV}$ [12]. In June 2016, intensive observations were triggered by high flux states of the source. Remarkably, the VHE flux was highest on the 13th and 14th of this month reaching $\sim 3$ Crab Unit (C.U.) ${ }^{1}$. We used two data sets from 13th and 14th June 2016 with effective time of about 137 minutes and 81 minutes, respectively, for MWL SED modeling. The zenith angle of the source is about $40^{\circ}$, and the energy threshold is $\sim 100 \mathrm{GeV}$ for such an angle [12].

We performed a detailed spectral analysis of the data during the highest VHE flux states. In addition to it, the intra-night variability was inspected. We made light curves above $300 \mathrm{GeV}$ and evaluated a characteristic time scale of the flux variations.

\footnotetext{
${ }^{1}$ flux level of the Crab Nebula under dark conditions measured above the same energy threshold [13]
} 


\subsection{Fermi Large Area Telescope}

The LAT is an instrument on board the Fermi Gamma-ray Space Telescope [14, 15]. It covers an energy range from a few tens of $\mathrm{MeV}$ to more than $300 \mathrm{GeV}$. It observes the whole sky every three hours during its standard survey mode. We focused on the data for 1.5 days from 12th June 21:00 to 14th June 9:00 in 2016, which are quasi-simultaneous with the MAGIC data on 13th and 14th June 2016. In order to keep statistics, we used the LAT data of this period for modeling both flares on 13th and 14th June 2016.

\subsection{Neil Gehrels Swift Observatory}

We used the public data of two instruments onboard Neil Gehrels Swift Observatory (hereafter Swift), namely, the X-ray telescope (XRT) and the UV/Optical Telescope (UVOT). For the spectral analysis at the major flares, we found observations in 13th and in 14th June 2016, for $\sim 70$ min and for $\sim 15 \mathrm{~min}$, respectively. These times are subsets of the MAGIC observation time.

The XRT is sensitive for $0.2-10 \mathrm{keV}$ [16]. The data were reduced and analyzed with the standard software xrtpipeline and XSPEC, respectively. We derived the differential energy flux from $0.6 \mathrm{keV}$ to $7.5 \mathrm{keV}$ for them.

The UVOT has six filters which have a narrow sensitive waveband from $170 \mathrm{~nm}$ to $600 \mathrm{~nm}$ $[17,18]$. For the simultaneous multi-waveband analysis on 13th and 14th June 2016, the data of the filters [W1, W2: centred at $192.8 \mathrm{~nm}$ ] and [W1, M1: centred at $224.6 \mathrm{~nm}, \mathrm{~W} 2]$ were available, respectively.

\section{Results}

\subsection{Spectra during the highest flux nights}

\subsubsection{Very-high-energy $\gamma$ ray}

A spectrum in the VHE $\gamma$ rays is quite important for this study because the high-energy peak of the SED is well spanned by the MAGIC observations. The $\gamma$-ray flux with energies above 300 $\mathrm{GeV}$ of $\sim 3.0$ C.U. and $\sim 2.8$ C.U. was observed on 13th and 14th June 2016, respectively. It is the highest level since 2002 and more than one order of magnitude higher than that during a typical low state. The SEDs in the VHE band on 13th and 14th June 2016 are very flat and extend beyond a few TeV. In both nights the EBL-corrected VHE spectra are better described with a curvature in the spectra rather than a simple power-law with no decisive preference among a log-parabola, a power-law with an exponential cutoff and a log-parabola with an exponential cutoff. Regarding any curved fit function amongst them, the local power-law index at $300 \mathrm{GeV}$ is harder than 2 around $300 \mathrm{GeV}$. These data and analysis enabled us to constrain the cutoff and peak energies with $\sim 10 \%$ and $\sim 20 \%$ statistical uncertainty, respectively. The SEDs peak at $\sim 0.4-0.7 \mathrm{TeV}$, and they have a cutoff above a few $\mathrm{TeV}$ when fitted with a power-law with a cutoff-type function.

\subsubsection{Other wavebands}

The fit of the LAT spectrum in the considered interval shows the power law index $1.56 \pm 0.20$. The index is marginally harder than the values reported in the 3FGL and the 4FGL catalogue, 
$1.88 \pm 0.02$ [23] and $1.82 \pm 0.01$ [24], respectively, by less than $2 \sigma$, although the fit ranges are different.

The spectra above $0.5 \mathrm{keV}$ extracted from the XRT data are well fitted by both a power-law and a log-parabola function. Fitting with a log-parabola does not improve the fit. The power law index is $1.81 \pm 0.01$ on 13th June and $1.82 \pm 0.01$ on 14th June 2016.

\subsection{Intra-night variability}

The investigation of intra-night variability in the VHE band is important to constrain the size of the emission region and to reproduce the physical conditions inside the source responsible for the second SED peak.

The VHE $\gamma$-ray flux exhibited fast variations for some nights in 2016, particularly for one of the nights with the highest VHE flux 13th June 2016. Based on a step in the VHE flux between two consecutive light curve bins, we quantised the shortest variability time scale as $36 \pm 14 \mathrm{~min}$ for 13th June 2016. Fitting individual substructures in the light curves with parametrized functions lead to similar results. The fast flux variability in the VHE band helped us to constrain the size of the emission region to be $R \leq 10^{15} \mathrm{~cm}$.

\subsection{Broadband emission modeling}

We have modelled the MWL SEDs during the flares on 13th and 14th June 2016 with three different theoretical frameworks considering one-zone leptonic, hadronic and lepto-hadronic models. A modified version of the code described in [19] was used for this.

\subsubsection{Leptonic model}

First we investigated a one-zone SSC model for the SEDs. The primary electron distribution is assumed to follow a broken power law described by two slopes. The break energy was determined by balancing the synchrotron cooling and the electron escape timescale. Under the assumption of spectral break due to radiative cooling, the electron spectral index after break was assumed to become softer by unity after the break.

The EBL-corrected VHE spectrum is very flat and extends up to several $\mathrm{TeV}$, especially for the 13th June flare. At such high energies, the inverse Compton component is generally suppressed because of fast radiative electron cooling and the Klein-Nishina effect. These constraints lead us to a conclusion that high values of Doppler factor and weak magnetic field is required for the simple SSC solutions. The MWL SED of 13th June 2016 can be explained with $\delta \geq 45-50$, whereas that of 14th June requires relatively smaller values of $\delta \geq 30$. It can be interpreted that a decrease in the jet Doppler factor lowered the spectral cutoff energy in the VHE data measured for 13th and 14th June 2016.

\subsubsection{Hadronic model}

As an alternative scenario, we investigated a model with relativistic protons injected into the emission region in addition to the primary leptons. The proton distribution is assumed to be a power law with exponential cutoff function. In the hadronic scenario, direct synchrotron radiation by protons can satisfactorily reproduce the SED peak located at few hundreds of $\mathrm{GeV}$. The lower energy peak is described by synchrotron radiation from the primary leptons. 
In this solution, the protons have to be accelerated up to few $\mathrm{EeV}$ energies. This is possible if the source has very high acceleration efficiency under magnetically dense environments. Strong magnetic fields of the order of $100 \mathrm{G}$ are required in the purely hadronic solutions to ensure fast radiative cooling of the protons in compliance with the rapid flux variability timescales measured from the source during 2016. The Doppler factors required for the hadronic solutions is smaller $(\delta \sim 25)$ than the values required for the leptonic models and represents more or less typical values for this class of sources.

\subsubsection{Lepto-hadronic model and implications for neutrino emission}

Generally the proton-synchrotron models predict neutrino fluxes lower than the sensitivity of present neutrino telescopes. For further investigations of possible neutrino emission, we studied a lepto-hadronic model for the flare on 13th June 2016 (since 14th June has a similar VHE flux level, the constraints on the level of neutrino emission would be same for this night as well). We assume an additional proton population with a power law spectrum defined by the same functional form as described in the hadronic modelling subsection, along with the relativistic electrons inside the emission region. In the lepto-hadronic solutions the second SED peak is explained by contributions from both the SSC component and the $p-\gamma$ cascade component (arising from synchrotron emission of secondary $e^{+} / e^{-}$pairs generated in photo-meson interations). The first SED peak is attributed to electron synchrotron, and the electron spectral indices and break Lorentz factors are comparable to those of the leptonic model. The magnetic field strength is $0.6 \mathrm{G}$, and an extremely strong field like the hadronic model is not required. In this case the required maximum proton energy is $\gamma_{p, \text { max }} \sim 6 \times 10^{7}$. The neutrino spectrum peak is mainly determined by the maximum proton energy. In the proton-synchrotron solutions, the inferred neutrino spectrum peaks at energies above few $\mathrm{EeV}$ in the observer frame and the flux at $0.1-100 \mathrm{PeV}$ is quite low. In case of the lepto-hadronic solution, the neutrino spectra peak about two orders of magnitude lower in energy because a much smaller value of the maximum proton energy is required.

From the lepto-hadronic solution, the integrated neutrino flux for $600 \mathrm{GeV}-100 \mathrm{TeV}$ is $\sim$ $5.5 \times 10^{-13} \mathrm{TeV} \mathrm{cm}^{-2} \mathrm{~s}^{-1}$. It is comparable to the upper limit flux for 1ES $1959+650$ obtained by IceCube [20]. Moreover, the lepto-hadronic solutions need very high values of jet power $\left(L_{j}>10^{48}\right.$ $\mathrm{erg} / \mathrm{s}$ ) exceeding the Eddington luminosity by more than 2 orders of magnitude and hence are energetically less favourable (see however [21]). These results imply that producing detectable neutrino emission during the 2016 and similar flares of 1ES 1959+650 is difficult.

\section{Acknowledgments}

MAGIC Collaboration: https://magic.mpp.mpg.de/acknowledgments_ICRC2019/

The Fermi LAT Collaboration acknowledges generous ongoing support from a number of agencies and institutes that have supported both the development and the operation of the LAT as well as scientific data analysis. These include the National Aeronautics and Space Administration and the Department of Energy in the United States, the Commissariat à l'Energie Atomique and the Centre National de la Recherche Scientifique / Institut National de Physique Nucléaire et de Physique des Particules in France, the Agenzia Spaziale Italiana and the Istituto Nazionale di Fisica Nucleare in Italy, the Ministry of Education, Culture, Sports, Science and Technology (MEXT), 
High Energy Accelerator Research Organization (KEK) and Japan Aerospace Exploration Agency (JAXA) in Japan, and the K. A. Wallenberg Foundation, the Swedish Research Council and the Swedish National Space Board in Sweden. Additional support for science analysis during the operations phase is gratefully acknowledged from the Istituto Nazionale di Astrofisica in Italy and the Centre National d'Études Spatiales in France. This work performed in part under DOE Contract DE-AC02-76SF00515.

We also appreciate a helpful framework which we used for the Fermi-LAT data analysis, Fermipy [22].

\section{References}

[1] E. S. Perlman et al., The Einstein Slew Survey Sample of BL Lacertae Objects, The Astrophysical Journal Supplement Series, 104, 251

[2] H. Krawczynski et al., Multiwavelength observations of strong flares from the TeV blazar 1ES 1959+ 650, The Astrophysical Journal, 601, 151

[3] G. Tagliaferri et al., Simultaneous multiwavelength observations of the blazar 1ES 1959+650 at a low TeV flux, The Astrophysical Journal, 679, 1029

[4] IceCube Collaboration et al., Multimessenger observations of a flaring blazar coincident with high-energy neutrino IceCube-170922A, Science, 361, eaat1378 [astro-ph/1807.08816]

[5] F. Halzen and D. Hooper, High energy neutrinos from the TeV Blazar 1ES $1959+650$, Astroparticle Physics, 23, 537-542 [astro-ph/0502449]

[6] K. S. Baliyan et al., Optical/NIR Observations of HBL 1ES 1959+625 from Mt Abu IR Observatory(MIRO), India, The Astronomer's Telegram, 9070, 1

[7] B. Kapanadze et al., A strong X-ray Flare in TeV blazar 1ES 1959+650, The Astronomer's Telegram, 8289,1

[8] B. Kapanadze et al., A new highest historical X-ray State in IES 1959+650, The Astronomer's Telegram, 9205, 1

[9] S. Buson et al., Fermi-LAT, FACT, MAGIC and VERITAS detection of increasing gamma-ray activity from the high-energy peaked BL Lac object IES 1959+650, The Astronomer's Telegram, 9010, 1

[10] A. Biland et al., Further increase of gamma-ray emission from the HBL 1ES 1959+650, The Astronomer's Telegram, 9148, 1

[11] A. Biland et al., FACT and MAGIC measure an increased gamma-ray flux from the HBL 1ES 1959+650, The Astronomer's Telegram, 9203, 1

[12] J. Aleksić et al., The major upgrade of the MAGIC telescopes, Part II: A performance study using observations of the Crab Nebula, Astroparticle Physics, 72, 76 - 94

[13] J. Albert et al., VHE $\gamma$-Ray Observation of the Crab Nebula and its Pulsar with the MAGIC Telescope, The Astrophysical Journal, 674, 1037

[14] W. B. Atwood et al., The Large Area Telescope on the Fermi Gamma-Ray Space Telescope Mission, The Astrophysical Journal, 697, 1071

[15] M. Ackermann et al., The Fermi Large Area Telescope on Orbit: Event Classification, Instrument Response Functions, and Calibration, The Astrophysical Journal Supplement Series, 203, 4 
[16] D. N. Burrows et al., The Swift X-Ray Telescope in X-Ray and Gamma-Ray Instrumentation for Astronomy XIII, 5165, 201-216

[17] P. W. A. Roming et al., The Swift ultra-violet/optical telescope, Space Science Reviews, 120, 95-142

[18] T. S. Poole et al., Photometric calibration of the Swift ultraviolet/optical telescope, Monthly Notices of the Royal Astronomical Society, 383, 627-645

[19] S. Ansoldi et al., The Blazar TXS 0506+056 Associated with a High-energy Neutrino: Insights into Extragalactic Jets and Cosmic-Ray Acceleration, The Astrophysical Journal Letters, 863, L10

[20] IceCube Collaboration et al., Search for steady point-like sources in the astrophysical muon neutrino flux with 8 years of IceCube data [hep-ph/1811.07979]

[21] M. V. Barkov et al. Rapid TeV Variability in Blazars as a Result of Jet-Star Interaction, The Astrophysical Journal,749, 119

[22] M. Wood, Fermipy: An open-source Python package for analysis of Fermi-LAT Data in proceedings of International Cosmic Ray Conference, PoS ( ICRC2 017 ) 824 (2017) [astro-ph/824]

[23] F. Acero et al., Fermi large area telescope third source catalog,The Astrophysical Journal Supplement Series, 218, 23 [astro-ph.HE/1501.02003]

[24] The Fermi-LAT collaboration, Fermi Large Area Telescope Fourth Source Catalog [astro-ph.HE/1902.10045] 